\title{
Bilateral Blindness Due to Acute Isolated Sphenoid Sinusitis- Is It Reversible? A Case Report
}

\author{
ROSHAN KUMAR VERMA, ${ }^{1}$ NARESH K PANDA ${ }^{1}$
}

\begin{abstract}
:
Acute isolated sphenoid sinusitis is a rare and is seen in only $3 \%$ of all cases of all sinusitis. It is frequently misdiagnosed because of its vague clinical presentation. We report a case of 35 year old female who presented to our emergency department with complaints of painless rapidly developing bilateral vision loss. Non contrast computed tomography of paranasal sinuses showed only mild opacification of sphenoid sinus. Endoscopic sphenoidectomy was performed within 4 hrs of presentation. After 48 hrs of surgery the patient vision returned to $6 / 6$ bilaterally. Acute sphenoid sinusitis should be considered in the differential diagnosis of acute vision loss. Awareness, early diagnosis, astute clinical sense and emergent intervention can prevent permanent complication.
\end{abstract}

Key words: Acute isolated sphenoid sinuitis, bilateral vision loss, endoscopic sphenoidotomy, reversible blindness.

\section{Introduction:}

Visual loss has been known to complicate bacterial sinusitis. ${ }^{1}$ It could be either due to direct spread of infection and inflammation or may be secondary to pressure on the exposed optic nerve. ${ }^{2}$ Spread of infection into orbital tissue is more common from ethmoid sinus due to its thin walls and to openings which communicate with the orbit and is usually assosciated with signs of orbital inflammation i.e. orbital pain, lid edema, orbital cellulitis, opthalmoplegia.

Isolated acute sphenoid sinusitis is an uncommon clinical entity and accounts for less than $3 \%$ of all cases of sinusitis. ${ }^{3}$ It is frequently misdiagnosed because of vague symptoms and paucity of clinical findings. Headache and visual loss is the most common. Rarely isolated acute sphenoid sinusitis could lead to bilateral acute vision loss. Permanent blindness is reported in $10 \%$ of cases of acute sphenoiditis. A high index of suspicion is necessary for early diagnosis and early intervention in such cases could lead to reversal of vision loss. A literature search showed very few case reports of reversible blindness following acute sphenoid sinusitis with prompt treatment. ${ }^{3}$

We report a case of 35-year-old female who presented to us with rapidly progressive bilateral vision loss with headache. Non contrast computed tomography of paranasal sinuses

1. Dr. Roshan Kumar Verma, Dr. Naresh K Panda, Department of Otolarngology and Head \& Neck surgery, Postgraduate Institute of Medical Education and Research, Chandigarh 160012. INDIA.

Correspondence : Dr. Roshan Kumar Verma, Assistant Professor, Department of Otolaryngology and Head \& Neck Surgery, Postgraduate Institute of Medical Education and Research, Chandigarh. INDIA - 160012. Fax : 91-172-2744401, Phone : 0091-0172-2747586 to 94 Ext.6764. E-mail: roshanverma@ hotmail.com. confirmed diagnosis of isolated acute sphenoid sinusitis. Emergency sphenoidotomy done resulted in reversal of vision loss bilaterally. This case is being presented to increase awareness among otolaryngologist about this condition develop high index of suspicion to prevent permanent complications due to sphenoid sinusitis.

\section{Case Report:}

A 35-year- old female presented to our Emergency department with complaints of rapidly progressing, painless, bilateral vision loss of vision. She initially complained of blurring of vision on right side for five days which progressed to complete loss of vision at time of presentation and history of blurring of vision on the left eye for 1 day. She complained of headache for 3 weeks. Headache was intermittent, sharp pricking type, bilateral fronto- temporal region $(\mathrm{R}>\mathrm{L})$, no diurnal variation and was relieved with medication. There was no history of Fever, nasal obstruction, nasal discharge, nasal bleed, trauma and nausea vomiting.

On examination anterior rhinoscopy showed mild deviation of septum to right side with congestion of posterior pharyngeal wall and postnasal drip. Eye examination showed no lid edema chemosis or conjunctival congestion. She had visual acuity of perception of hand movements close to face on right eye with reactive afferent pupillary defect and visual acuity on left eye was 6/60 with normal size and normal reacting pupil. Extra ocular movements were full and free in both eyes. Fundus examination showed normal optic disc on both sides.

Non contrast enhanced computed tomography of paranasal sinus was done which showed heterogeneous opacity filling the sphenoid sinus with bony erosion and destruction of roof and dorsum sellae. Rest of others paranasal sinuses 
were normal (Fig 1). Magnetic resonance imaging showed mucosal thickening in the sphenoid sinus suggestive of inflammatory pathology. (Fig.-2)

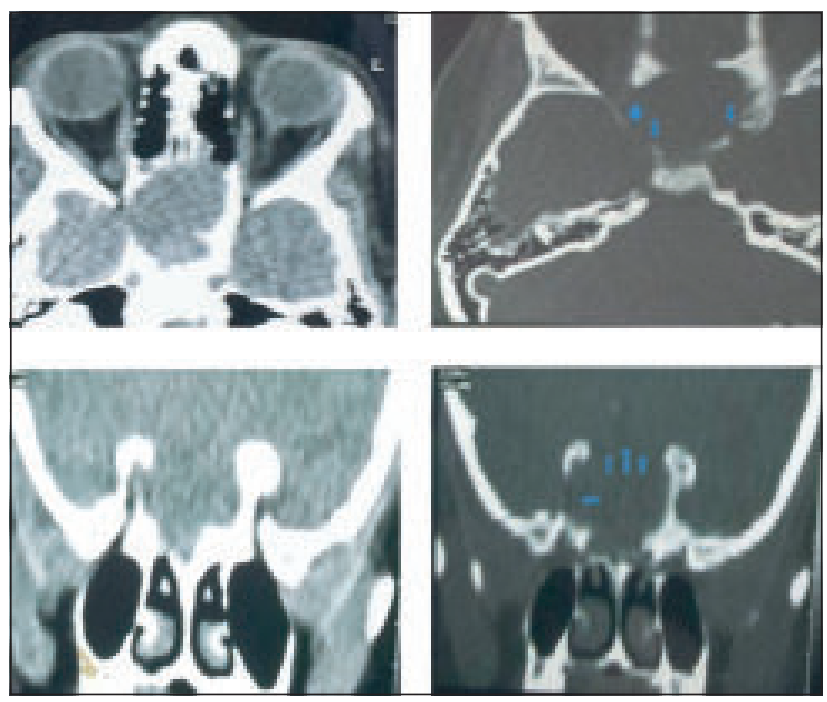

Fig - 1: Computed tomography of nose and paranasal sinus (coronal and axial cuts) showing heterogeneous opacity of sphenoid sinus with erosion and destruction of lateral wall and roof of sphenoid sinus (shown by arrow)

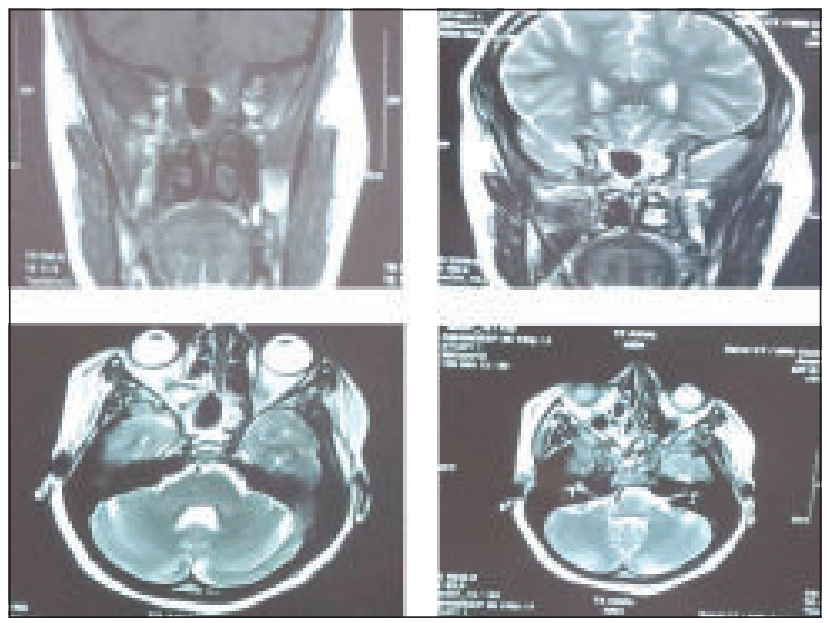

Fig - 2: MRI (coronal and axial cuts) showing mucosal soft tissue enhancement in the sphenoid sinus suggestive of inflammatory sinus disease.

Treatment was started with intravenous amoxicillin and clavulinic acid and Metonidazole and cloxacillin and patient was taken up for endoscopic shenoidotomy in emergency. Pinkish polypoidal tissue was filling the sphenoid sinus and obstructing the sphenoid ostium. Polypoidal tissue was removed from the sphenoid sinus and sent for fungal smear and culture and for gram stain and culture.
Postoperatively after 48hours of surgery, the patient's vision returned to $6 / 6$ bilaterally. She was continued on intravenous antibiotics for one week and discharged. She is on regular follow up and is asymptomatic at present.

\section{Discussion:}

Von Alyea has describes sphenoid sinus as one of the most neglected sinus. ${ }^{4}$ It is lined by pseudostatified epithelium and has fewer mucus secreting cells, it is associated with fewer drainage problems and so lesser incidence of sphenoid sinusitis. Sphenoid sinus is in close relation with various vital structures like cranial nerves II to VI, internal carotid artery, cavernous sinus, pituitary gland spenopalatine nerve and ganglion and dura. Optic nerves may pass through the sphenoid sinus or may be closely related to sphenoid sinus in $10 \%$ cases. $^{5}$ Optic canal may be dehiscent in $4 \%$ cases. $^{6}$ These anatomical variants of sphenoid sinus and the abnormal location and size of sphenoid ostium, bony dehiscence over optic canal increase the risk of visual complaints sphenoid in sinus diseases.

Isolated sphenoid sinus disease is rare. Hnatuk et al suggested incidence of isolated sinus disease to be less than $1 \%$ of all sinus disease. ${ }^{7}$ Isolated acute sphenoid sinusitis is even rarer and accounts for $3 \%$ of all cases of sinusitis. ${ }^{3}$ Vague symptomatology and paucity of clinical findings delay the diagnosis until patient develops neurological complications. An astute clinical sense is required to make early diagnosis and prevent complication.

Isolated acute sphenoid sinusitis most commonly present with headache in the vertex region. It may sometimes interfere with sleep. Visual disturbance is the second common symptom of isolated acute sphenoid sinusitis and may range from diplopia, blurred vision to blindness. Blindness is usually irreversible and only few cases in the literature exist where the blindness was reversible. $6,8,9,10$ Our case is one amongst such rare cases reported in the literature.

Vision loss in isolated acute sphenoid sinusitis may be due to various reasons. Inflammation of the optic nerve due to direct spread of infection in the presence of optic canal dehiscence can lead to optic neuritis and blindness. ${ }^{11}$ Ischemia due to venous occlusion or pressure from orbital edema or abscess can lead to central venous occlusion and blindness. Direct compressive effect over the dehiscent optic nerve or optic chiasma can also lead to blindness. ${ }^{12}$ In our case computed tomographic scan showed erosion of the roof of sphenoid sinus. We suspect that pressure over the optic chiasma could have lead to blindness.

Does the blindness following acute sphenoiditis reversible? A literature search showed very few case reports of blindness 
which was reversed by prompt treatment. ${ }^{6,8,9,10,12}$ The return of blindness following acute sphenoid sinusitis depends on many factors. Prompt surgical management before irreversible changes occur and the degree of visual loss are important factors having prognostic significance. Our patient had no signs of orbital inflammation or optic nerve changes, hence prompt surgical intervention caused the reversal of blindness seen in our case.

Isolated sphenoid sinusitis with visual complication requires prompt surgical intervention. Endoscopic sphenoidotomy is the best surgical approach for opening and drainage of sphenoid sinus and if required can be combined with decompression of optic nerve to release the pressure on the nerve. ${ }^{13,14}$ Surgery should be directed at removing the purulent material, obtaining cultures, removing irreversibly diseased mucosa, and maintaining drainage of the sphenoid sinus. Intravenous antibiotics and steroid therapy post operatively must be continued. ${ }^{15}$ The incidence of permanent blindness in acute sphenoiditis is reported to be $10.5 \%$. An early diagnosis and prompt surgical intervention can prevent complication of permanent blindness in cases of acute sphenoid sinusitis.

\section{Conclusion:}

Isolated sphenoid sinusitis is rare and frequently misdiagnosed. The most common presenting signs and symptoms include headache, visual loss and cranial nerve palsies. Astute clinical sense and early diagnosis and prompt surgical management can prevent permanent blindness as we found out in our case.

\section{Conflict of interest: None.}

\section{References:}

1. Tarazi AE, Shikani AH. Irreversible unilateral vision loss due to acute sinusitis. Arch Otolaryngol Jead and Neck Surg.1991; 117: 1400-1401.

2. Chandler J, Langenbruner D J, Stevens ER. The pathogenesis of orbital complications in sinusitis. Laryngoscope.1970; 80: 1414-1428.
3. Lew D, Southwick FS, Montogomerry WW, Weber AL, Baker AS. Sphenoid sinusitis; a review of 30 cases. N Engl J Med. 1983; 309: 1149-1154.

4. Van Aleya OE. Sphenoid sinus: anatomic study with consideration of clinical significance of the structural characteristics of sphenoid sinus. Arch Otolaryngol.1941; 34: $225-253$.

5. De Lano MC, Fun FY and Zinreich SJ. Relationship of optic nerve to Posterior paranasal sinus. A CT anatomic study. American journal of neuroradiology 17: 669-675.

6. Postma G, Chole RA, Nemzek WR. Reversible blindness due to acute sphenoiditis. Otolaryngol Head and Neck Surgery. $1995 ; 112$ : 742-746.

7. Hnatuk LA, MacDonald RE, Papsin BC. Isolated sphenoid sinusitis- the Toronto hospital for sick children experience and review of literature. J otolaryngol 1994; 23: 36-41.

8. Lisa TG, Soly B, Joseph M, Frohman L. Visual loss reversed after treatment of acute bacterial sinusitis. Laryngoscope 1996; 106: 148-151.

9. Shawn CR, Elizabeth ME. Acute sphenoid sinusitis induced blindness: A case report. The Journal of Emergency Medicine. 2012; 43: 123-124.

10. Rohilla S, Yadav RK, Rawal M et al. An unusual case of bilateral reversible blindness in a child: crucial role of imaging. Journal of Pediatric Neurology. 2011; 9: 501-504.

11. Salvin ML, Glaser JS. Acute severe irreversible visual loss with sphenoethmoiditis-posterior orbital cellulitis. Arch opthalmol.1987; 97: 345-348.

12. Dale BAB, Mackenzie IJ. The complications of sphenoid sinusitis. J Laryngol otol.1983; 97: 661-670.

13. Pitkaranta A, Atula T, Lindahl P, Saxen H, Malmberg H. Unilateral blindness in a child with acute sinusitis. Rhinology 2000; 38: 43-4.

14. Patt B, Manning C. Blindness resulting from orbital complications of sinusitis. Otolaryngol Head Neck Surg 1991; 104: 789-95.

15. Tomac S, Turgut S. Orbital cellulitis and irreversible visual loss owing to acute sinusitis. Ann Ophthalmol 2006; 38: 131-3. 